\title{
EFFECT OF MUSIC THERAPY INTERVENTION ON PHYSICAL FUNCTIONS AND MENTAL HEALTH OF PATIENTS WITH ANKYLOSING SPONDYLITIS
}

\author{
Yuan Qin \\ Conservatory of Music, Suzhou University, Suzhou, China
}

received: 17.4.2020;

revised: 21.8.2020;

accepted: 31.8 .2020

\section{SUMMARY}

Background: This study aims to investigate the influence of traditional Chinese music on the physical and psychological functions of hospitalized patients with ankylosing spondylitis $(A S)$.

Subjects and methods: A total of 120 patients with AS admitted to the People's Hospital of Anhui Province between March 2018 and March 2019 were randomly divided into experiment and control groups. The experiment group was a traditional music group composed of 56 patients, namely, 50 males and 6 females. The control group was further divided into a painting group and a routine treatment group with a total of 64 patients, namely, 48 males and 16 females. The physical and psychological functions of each group of patients before and after the intervention were assessed in terms of physical and psychological function dimensions obtained before and after the intervention.

Results: After 8 weeks of treatment, the score in terms of physical functions is the highest in the music group $(P<0.05)$, and the difference in eating functions is statistically significant $(P<0.001)$. This finding suggests that the music group is better than the painting group and the routine treatment group $(P<0.05)$. In terms of the score in sexual functions, the following trend is observed: music group $>$ painting group $>$ routine treatment group. The difference in their psychological functions is statistically significant $(P<0.001)$. In terms of the score in psychological functions, the following trend is obtained: music group $>$ painting group $>$ routine treatment group. The three groups significantly differ in their scores in mental tension, negative emotion, positive emotion, cognitive functions, and self-esteem. All of these dimensions show the following pattern: music group>painting group $>$ routine treatment group. Cross-group comparisons between the three groups are also statistically significant $(P<0.05)$.

Conclusions: Traditional Chinese music therapy and painting therapy can promote the recovery of patients' physical and psychological functions. Traditional Chinese music intervention therapy is better than painting therapy and routine hospitalization in promoting the recovery of physical and psychological functions of hospitalized patients.

Key words: mental health - music therapy - ankylosing spondylitis - physical functions

\section{INTRODUCTION}

Ankylosing spondylitis (AS) is a chronic and progressive rheumatic disease characterized by inflammation, especially sacroiliitis (Kjeken et al. 2013). The main clinical features of AS are inflammatory back pain, joint stiffness, and fatigue, which are not easily relieved. These symptoms often lead to structural and functional impairment in the general health and physical functions of an individual (Dundar et al. 2014, Zagar et al. 2019). Hakkou et al. (2013) determined that this disease occurs mostly in young males aged $20-30$ years. For males in adolescence, the decline and loss of physical functions cause various health problems, such as insomnia and anxiety. Y1lmaz et al. (2013) considered that AS also affects the mental health of patients and reduce their sense of well-being. Wu et al. (2017) revealed that the decline in the mental health level negatively affects the physical health and recovery of patients with AS. Therefore, to improve the physical functions and mental health of patients with AS, clinical psychologists proposed different intervention methods from various theoretical perspectives. In general, medication intervention, psychological counseling interven- tion, and other approaches have been developed. Medication intervention often improves a patient's mental health through a particular treatment. For psychotherapy intervention, O'Dwyer et al. (2017) increased the motivation and frequency of the participation of patients with AS in physical exercise through a one-to-one semistructured consultation, thereby reducing their anxiety and depression levels. From the perspective of intervention implementation, a family-based intervention and another intervention that focuses on patients' selfmanagement have been established (Spencer et al. 2015).

Music intervention has been used extensively in clinical applications, and related studies have shown that music intervention works efficiently in reducing the degree of pain (Bradt et al. 2016), trait anxiety (Lee et al. 2017), and depression in patients (Fancourt et al. 2016). Garza-Villarreal et al. (2017) emphasized that music is a non-aggressive and low-cost intervention easily accepted by patients. They conducted a metaanalysis on 768 articles on the relationship of music with the physical and mental health of patients with chronic pain. Their results show that music can alleviate chronic pain and depression, especially those observed in patients who actively choose music intervention. 
Although the effect of music intervention on chronic pain diseases has been confirmed, related intervention studies have not included AS, a typical chronic pain disease. In China, although many discussions and studies on the effect of music therapy on alleviating the physiological pain of patients with cancer or other patient groups (Xue et al. 2017, Zeng et al. 2015), cases in which music therapy is applied to patients with AS have yet to be presented. Based on previous studies, music intervention therapy for the physical functions and mental health of patients with AS is innovatively used in the present study to expand the application of music intervention. The combination of music intervention and other psychological behavioral interventions can further enrich the intervention for patients with AS, providing reference methods and detailed criteria for other researchers and clinical psychologists.

\section{SUBJECTS AND METHODS}

\section{Participants}

A total of 120 patients with AS admitted to the People's Hospital of Anhui Province between March 2018 and March 2019 were randomly divided into two groups. The experiment group (music intervention) comprised 56 patients, namely, 50 males and 6 females, aged 18-42 years with an average age of $26.6 \pm 4.5$ years. Their disease duration ranged from 3 months to 15 years with an average of $4.6 \pm 0.7$ years. The control group included 64 patients, namely, 48 males and 16 females, aged 17-41 years with an average age of $25.3 \pm 5.2$ years. Their disease duration ranged from 3 months to 16 years with an average of $4.7 \pm 0.2$ years. The control group was further divided into a routine treatment group and a painting group. The two groups did not significantly differ in the following clinical data: gender, age, and disease duration $(\mathrm{P}>0.05)$. This observation suggested that they were comparable. The details are shown in Table 1.

One-way ANOVA shows no significant difference in age between the groups $(\mathrm{F}=0.606, \mathrm{P}=0.547>0.05)$. Chisquare test indicates no statistically significant difference in gender distribution between the three groups $(2=4.888, \mathrm{P}=0.106>0.05)$. Kruskal-Wallis $\mathrm{H}$ test reveals no significant difference in the distribution of disease duration between the three groups $(2=0.539, \mathrm{P}=0.764)$. The general information of the three groups is comparable.

\section{Methods}

The routine treatment group was given $15 \mathrm{mg}$ of oral meloxicam tablets twice daily before bedtime. Meloxicam was supplemented by routine psychological intervention and counseling in the hospital. After the patients were discharged, the patients followed routine discharge instructions and were tracked weekly to know their recovery.

The painting group started painting practice within the 1 st week in accordance with routine treatment in the hospital and continued to receive routine treatment in the hospital. The patients who participated in painting therapy were grouped with no more than 10 members in each group and received painting therapy four times a week. The treatment lasted 60 minutes in each time. Group treatment and individual guidance were combined. Painting activities were conducted in a clean, quiet, and bright painting room. The patients learned painting and calligraphy in accordance with copybooks and painting tutorials. The investigators first observed, guided, and inspired the patients to innovate. They then displayed the patients' finished works in the painting room. Then, the investigators and the patients participated in the evaluation of outstanding works and gave rewards. A telephone follow-up was conducted once a week after discharge to trace the patients' insistence on painting intervention.

Traditional Chinese music intervention was conducted in the experiment group based on the control group. A number of traditional Chinese music songs, namely, "Spring is coming," "Beaming with joy," "Ah, no worries," and "Making progress," were selected to develop personalized music packages suitable for the patients in accordance with the patients' physical and psychological symptoms. Personalized music packages are developed in accordance with the categories of traditional Chinese music. According to 4 categories of traditional Chinese music, they are divided into 4 types of music packages, that is, court music package, poet music package, religious music package and folk music package respectively. The selection criterion of music is recommended by www.9ku.com, a relatively authoritative platform in China. The music package was placed in the patient's phone. The music therapy was provided to the patients throughout the whole hospitalization process. The nurse-in-charge guided the patient to listen for 30 minutes in the morning and before going to bed.

Table 1. Cross-group comparison of general data

\begin{tabular}{lccccc}
\hline \multirow{2}{*}{ Factor } & \multicolumn{2}{c}{ Control group } & \multirow{2}{*}{ Music group } & \multirow{2}{*}{$\mathrm{F} / \chi^{2}$} & \multirow{2}{*}{$\mathrm{P}$} \\
\hline Gender & Routine treatment group & Painting group & & & \\
$\quad$ & & & & 4.888 & 0.106 \\
Male & $23(71.88 \%)$ & $25(78.13 \%)$ & $50(89.29 \%)$ & & \\
Female & $9(28.13 \%)$ & $7(21.88 \%)$ & $6(10.71 \%)$ & \\
Age & $25.16 \pm 5.66$ & $26.28 \pm 5.34$ & $26.36 \pm 4.77$ & 0.606 & 0.547 \\
Disease duration & $4.81 \pm 3.4$ & $4.5 \pm 3.23$ & $5.06 \pm 3.57$ & & \\
Median duration & $3.5(2-7)$ & $3.5(2-5.5)$ & $4(3-7)$ & 0.539 & 0.764 \\
\hline
\end{tabular}


Depending on personal preference, the patient could also listen at any time, and the course of treatment lasted 10 days. The treatment volume is $20-40 \mathrm{~dB}$. Similarly, the connotation and background of the music were introduced during the treatment process, and the patient was guided into the artistic conception to increase the effect. Every week, the nurse communicated with the patient once, encouraged the patient to express inner feelings, positively evaluated each practice result, focused on the practice of collective positive psychology, and conducted a telephone follow-up every week after discharge to check whether the patient adhered to music intervention. The patients were tested through GQO-LI-74 before and after eight courses of treatment to evaluate the intervention effect on their physical and psychological functions.

\section{Diagnostic criteria}

Our diagnostic criteria were based on Clinical Diagnosis and Treatment Guide proposed by the Chinese Medical Association for the diagnosis of AS.

\section{Inclusion criteria and exclusion criteria}

The following inclusion criteria were considered:

- patients with diagnosed AS;

- patients with clear consciousness and without disturbance to participate in the investigation;

- patients who were willing to participate in this study and given informed consent;

- patients with a certain level of education;

- patients who knew and understood the content of traditional Chinese music therapy (which originated from the long-standing traditional culture background of China, and the unique traditional Chinese culture gave birth to the unique traditional music.

It was handed down over time, rather than created contemporarily. The traditional Chinese music therapy in this study is to assist patients in selecting music packages that they are interested in and give music interventions by means of listening) and were willing to cooperate. The following exclusion criteria were considered:

- patients with severe hearing or mental retardation and were unwilling to receive treatment or observation;

- patients with mental disorder or were unable to cooperate with the researcher.

\section{Measures}

Generic Quality of Life Inventory (GQO-LI-74) (Chen et al. 2007) was used in this study. This inventory consists of 20 factors with 74 items, and each item is quantified from very poor ( 1 point) to very good ( 5 points). Each factor is scored a maximum of 20 points and a minimum of 4 points. In particular, the physical and psychological functions in the inventory consist of sleep and energy, physical discomfort, and sensory functions; the higher the scores in eating functions, psychological functions, physical functions, sexual functions or exercise, and sensory functions are, the better the recovery of physical and psychological functions is. The test-retest reliability of the inventory is $0.84-0.93$, and the internal consistency reliability is $0.66-0.69$. The inventory has good content validity, construct validity, and criterion validity.

\section{Statistical Analysis}

Data were statistically analyzed using SPSS 24.0. Mean \pm standard deviation was used to describe the distribution of normal distribution quantitative data. One-way ANOVA was performed to compare differences between groups. LSD-t was conducted to compare the two groups when the overall difference was significant. Median and quartile were used to describe the distribution of non-normal distribution quantitative data. Kruskal-Wallis $\mathrm{H}$ test was carried out to compare the differences between the groups. Frequency and composition ratio were determined to describe the distribution of qualitative data. Chi-square test was employed to compare the differences between the groups. All of the tests in this study were two sided, and results were considered statistically significant at $\mathrm{P}<0.05$.

\section{RESULTS}

\section{Comparison of physical and psychological functions between groups before enrollment}

In Table 2, no statistically significant differences in physical and psychological functions are observed between the three groups before enrollment $(\mathrm{P}>0.05)$. No statistically significant differences in physical functions are found between the three groups $(\mathrm{F}=0.874, \mathrm{P}=0.420)$. Similarly, the groups do not significantly differ in terms of sleep and energy, physical discomfort, eating functions, sexual functions, exercise, and sensory functions (the corresponding $\mathrm{F}$ is $0.072,1.010,0.031,1.242$, and 0.058 , respectively. Their corresponding $\mathrm{P}$ values are $0.931,0.367,0.970,0.293$, and 0.944 , respectively, which are $>0.05$. The psychological functions between the three groups do not significantly differ $(\mathrm{F}=0.755$, $\mathrm{P}=0.472$ ). The groups do not also significantly vary in terms of mental tension, negative emotion, positive emotion, cognitive functions, and self-esteem (their corresponding $\mathrm{F}$ values are $0.436,0.503,0.272,1.645$, and 0.302 , respectively; their corresponding $P$ values are $0.648,0.606,0.762,0.197$, and 0.740 , respectively, which are $>0.05$ ). Hence, the physical and psychological functions of the three groups are comparable before enrollment.

\section{Comparison of physical and psychological functions between groups after 8 weeks of treatment}

Tables 3 and 4 show that the difference in physical functions between the three groups is statistically significant after 8 weeks of treatment $(\mathrm{F}=164.071, \mathrm{P}<0.001)$. 
Table 2. Comparison of physical and psychological functions between groups before enrollment

\begin{tabular}{lccccc}
\hline Dimension & Routine treatment group & Painting group & Music group & F & P \\
\hline Physical functions & $36.48 \pm 6.14$ & $36.56 \pm 5.79$ & $34.46 \pm 6.13$ & 1.734 & 0.181 \\
Sleep and energy & $9.63 \pm 2.59$ & $9.41 \pm 2.2$ & $9.57 \pm 2.49$ & 0.072 & 0.931 \\
Physical discomfort & $9.72 \pm 2.37$ & $10.16 \pm 2.4$ & $9.45 \pm 2.1$ & 1.010 & 0.367 \\
Eating functions & $10.09 \pm 2.9$ & $10.03 \pm 2.75$ & $9.95 \pm 2.65$ & 0.031 & 0.970 \\
Sexual functions & $8.81 \pm 1.89$ & $8.88 \pm 2.3$ & $7.75 \pm 2.25$ & 3.787 & 0.025 \\
Exercise and sensory functions & $10.94 \pm 2.03$ & $10.78 \pm 1.64$ & $10.86 \pm 1.82$ & 0.058 & 0.944 \\
Psychological functions & $31.88 \pm 5.33$ & $31.33 \pm 4.88$ & $30.56 \pm 4.81$ & 0.755 & 0.472 \\
Mental tension & $8 \pm 1.63$ & $8.28 \pm 1.73$ & $7.95 \pm 1.63$ & 0.436 & 0.648 \\
Negative emotion & $8.19 \pm 1.73$ & $7.81 \pm 1.67$ & $8.16 \pm 1.77$ & 0.503 & 0.606 \\
Positive emotion & $8.81 \pm 2.19$ & $8.44 \pm 1.29$ & $8.61 \pm 2.28$ & 0.272 & 0.762 \\
Cognitive functions & $10.38 \pm 1.96$ & $10.09 \pm 2.08$ & $9.63 \pm 1.83$ & 1.645 & 0.197 \\
Self-esteem & $10.13 \pm 2.15$ & $10.44 \pm 1.97$ & $10.11 \pm 1.98$ & 0.302 & 0.740 \\
\hline
\end{tabular}

Table 3. Comparison of physical and psychological functions between groups after 8 weeks of treatment

\begin{tabular}{lcccrc}
\hline Dimension & Routine treatment group & Painting group & Music group & $\mathrm{F}$ & $\mathrm{P}$ \\
\hline Physical functions & $45.66 \pm 5.01$ & $58.87 \pm 6.04$ & $68.82 \pm 6.04$ & 164.071 & $<0.001$ \\
Sleep and energy & $11.63 \pm 2.41$ & $14.44 \pm 2.2$ & $16.59 \pm 1.99$ & 53.979 & $<0.001$ \\
Physical discomfort & $10.5 \pm 2.05$ & $12.34 \pm 1.84$ & $14.61 \pm 2.42$ & 37.567 & $<0.001$ \\
Eating functions & $13.78 \pm 2.43$ & $14.88 \pm 2.32$ & $16.21 \pm 2.46$ & 10.718 & $<0.001$ \\
Sexual functions & $8.66 \pm 1.66$ & $10.16 \pm 2.03$ & $11.21 \pm 1.59$ & 22.155 & $<0.001$ \\
Exercise and sensory functions & $11.97 \pm 1.79$ & $15.28 \pm 1.63$ & $16.43 \pm 1.57$ & 75.684 & $<0.001$ \\
Psychological functions & $41.29 \pm 7.13$ & $60.98 \pm 6.39$ & $69.02 \pm 4.62$ & 229.535 & $<0.001$ \\
Mental tension & $10.34 \pm 2.16$ & $13.53 \pm 1.68$ & $14.89 \pm 1.84$ & 59.182 & $<0.001$ \\
Negative emotion & $10.75 \pm 1.92$ & $13.81 \pm 1.86$ & $14.79 \pm 1.52$ & 56.668 & $<0.001$ \\
Positive emotion & $10.06 \pm 2.2$ & $14.28 \pm 2.54$ & $15.39 \pm 1.79$ & 66.170 & $<0.001$ \\
Cognitive functions & $11.31 \pm 2.05$ & $14.56 \pm 2.03$ & $15.63 \pm 1.7$ & 53.858 & $<0.001$ \\
Self-esteem & $10.56 \pm 1.9$ & $12.59 \pm 2.42$ & $14.52 \pm 1.94$ & 37.955 & $<0.001$ \\
\hline
\end{tabular}

Table 4. Comparison of physical and psychological functions between the two groups after 8 weeks of treatment

\begin{tabular}{lccc}
\hline \multirow{2}{*}{ Dimension } & \multicolumn{2}{c}{ P of comparison between two groups } \\
& $\begin{array}{c}\text { Routine treatment group } \\
\text { vs painting group }\end{array}$ & $\begin{array}{c}\text { Routine treatment group } \\
\text { vs music group }\end{array}$ & $\begin{array}{c}\text { Painting group } \\
\text { vs music group }\end{array}$ \\
\hline Physical functions & $<0.001$ & $<0.001$ & $<0.001$ \\
Sleep and energy & $<0.001$ & $<0.001$ & $<0.001$ \\
Physical discomfort & 0.001 & $<0.001$ & $<0.001$ \\
Eating functions & 0.073 & $<0.001$ & 0.014 \\
Sexual functions & 0.001 & $<0.001$ & 0.007 \\
Exercise and sensory functions & $<0.001$ & $<0.001$ & 0.002 \\
Psychological functions & $<0.001$ & $<0.001$ & $<0.001$ \\
Mental tension & $<0.001$ & $<0.001$ & 0.002 \\
Negative emotion & $<0.001$ & $<0.001$ & 0.012 \\
Positive emotion & $<0.001$ & $<0.001$ & 0.020 \\
Cognitive functions & $<0.001$ & $<0.001$ & 0.012 \\
Self-esteem & $<0.001$ & $<0.001$ & $<0.001$ \\
\hline
\end{tabular}

In terms of the score in physical functions, the following trend is observed: music group $>$ painting group $>$ routine treatment group. No statistically significant difference is found between the two groups $(\mathrm{P}<0.05)$. The difference in sleep and energy between the three groups is statistically significant $(\mathrm{F}=53.979, \mathrm{P}<0.001)$. In terms of the score in sleep and energy, the following trend is observed: music group $>$ painting group $>$ routine treatment group $(\mathrm{P}<0.05$ in the comparison between the two groups). The difference in physical discomfort between the three groups is statistically significant $(\mathrm{F}=37.567$, $\mathrm{P}<0.001)$. 
The best scores are detected in the music group, followed by the painting group and then the routine treatment group $(\mathrm{P}<0.05$ in comparison between the two groups). The difference in eating functions between the three groups is statistically significant $(\mathrm{F}=10.718, \mathrm{P}<0.001)$. In terms of the score in eating function, the following trend is observed: music group $>$ painting group $>$ routine treatment group. The comparison between the two groups shows no statistically significant difference between the routine treatment group and the painting group $(\mathrm{P}=0.073)$, whereas the music group is better than the painting group and the routine treatment group $(\mathrm{P}<0.05)$. The difference in sexual functions between the three groups is statistically significant $(\mathrm{F}=22.155, \mathrm{P}<0.05)$. In terms of the score in sexual functions, the following trend is observed: music group $>$ painting group $>$ routine treatment group $(\mathrm{P}<0.05$ in the comparison between the two groups). The difference in exercise and sensory functions between the three groups is statistically significant $(\mathrm{F}=75.684, \mathrm{P}<0.001)$. In terms of the score in exercise and sensory functions, the following trend is observed: music group $>$ painting group $>$ routine treatment group $(\mathrm{P}<0.05$ in the comparison between the two groups). The difference in psychological functions between the three groups is statistically significant $(\mathrm{F}=229.535, \mathrm{P}<0.001)$. In terms of the score in psychological functions, the following trend is obtainned: music group $>$ painting group $>$ routine treatment group. The two groups significantly differ $(\mathrm{P}<0.05)$. The three groups significantly vary in terms of the factors, such as mental tension, negative emotion, positive emotion, cognitive functions, and self-esteem. The corresponding $\mathrm{F}$ values are 59.182, 56.668, 66.170, 53.858 and 37.955 , respectively $(\mathrm{P}<0.001$ in all cases). In all of the factors, the following trend is detected: music group $>$ painting group $>$ routine treatment group. The two groups significantly differ $(\mathrm{P}<0.05)$.

\section{DISCUSSION}

The experiment group and the control group do not significantly vary before intervention in terms of the recovery effect or the mental health level. After 8 weeks of intervention, the recovery and quality of life are significantly improved in the painting group and the music group based on routine treatment therapy compared with the pre-intervention level and the routine treatment group. Thus, music therapy and painting therapy have a good effect on promoting the recovery and quality of life of patients with AS.

First, music therapy can promote the recovery and quality of life of patients AS. The result is consistent with the finding of Zhang et al. (2018), who used the Chinese five-element music therapy to improve the mental health of patients with advanced malignant tumors. Therefore, music therapy improves the mental health level of patients to a certain extent. Music therapy can improve the physical and mental health of patients possibly because of the following paths. First, music therapy can affect patients' emotions (Koelsch 2015). Musical melody can influence people's emotions, which are key factors influencing individuals' cognition, decision-making, and actions (Koelsch 2018). Music can influence individuals' cognitive evaluation process. Those who are exposed to positive music are more positive about things. For patients, listening to positive music can increase their attention to the positive aspects of things, thus improving their confidence in recovery (Hou 2017). Similarly, positive and enjoyable music can also help reduce individuals' negative experiences of diseases, such as fear, worry, and fear (Carlson et al. 2015). Second, music therapy can influence patients' psychology (Gallego \& Garcia 2017). Positive music may create an emotional infection that improves patients' ability to work and remember, and studies have shown that emotion-related memory has a long-lasting effect on individuals (Tumuluri et al. 2017). Third, music therapy can also directly promote individuals' behaviors. According to the Heath Action Process Approach (HAPA) model, positive music enhances the individual's self-efficacy in implementting healthy behaviors, whereas self-efficacy increases and directly affects the internal impetus that produces healthy behaviors (Ghetti 2011).

Second, painting therapy can promote the physical and mental health level of patients to some extent. The result is consistent with those obtained by Zhao et al. (2011), who used painting therapy and yoga therapy for patients with schizophrenia. Painting therapy is better than routine treatment in improving the patients' quality of life. The present result is consistent with previous studies on art therapy (Van Lith 2016). Freud's catharsis theory believes that individuals can only maintain their mental health by excreting negative emotions, such as internal pain and dissatisfaction, whereas painting is the way people express themselves. People use painting to express inner feelings and cognition, thereby venting negative feelings and improving mental health; correspondingly, mental health also positively affects physical health. Thus, painting therapy can also improve the physical and mental health levels of patients to some extent (Yang et al. 2017, Li \& Ren 2019).

According to the results of this study, after 8 weeks of intervention, the physical functions of the objects in the music group and the painting group are improved compared with those of the routine treatment group. However, the comparison results show that the physical functions of the music group are significantly higher than those of the painting group. Hence, music therapy has the best intervention effect on the recovery of the physical functions of patients with AS. Specifically, first, music therapy has the best effect on the intervention for the sleep and energy of patients with AS. The result is consistent with the conclusion drawn by Wang et al. (2014), who stated that music therapy positively affects patients with severe and 
chronic insomnia. Our finding is also consistent with the meta-analysis results obtained by De Niet et al. (2009), who determined that music-related relaxation activities can improve sleep quality. Relaxing music can activate the processing in the corresponding areas of the brain, thereby changing individuals' cognitive and emotional experiences, whereas positive emotions are good medicines that help patients fall asleep quickly (Thompson 2015). Second, music therapy reduces the physical discomfort of patients with AS to the greatest degree. This result is consistent with the finding of $\mathrm{Wu}$ (2017). Wu (2017) also divided 27 college students into two groups: routine intervention and music therapy intervention. Their results show that the relaxation of the group subjected to music therapy intervention is significantly improved, and pain is significantly reduced. Kekecs et al. (2013) proposed in their meta-analysis that music therapy is a positive way to reduce physical discomfort possibly because individuals immersed in music pay less attention to physical discomfort. Third, music therapy has the best intervention effect on the feeding functions of patients with AS. This result is consistent with the findings of Loewy et al. (2013), who used music to treat a patient with anorexia nervosa, effectively reducing the patient's clinical symptoms. Music therapy can promote the satisfaction of patients' basic psychological needs. In particular, patients improve the satisfaction of their needs for independence, belonging, and competence during music therapy process, thus reducing psychological stress and alleviating eating difficulties (Bibb et al. 2015). Fourth, music therapy has the best effect on improving the sexual functions of patients with AS. Robarts (2003) used music therapy as intervention for a child who is a survivor of early trauma and sexual abuse, and the effect is quite clear, confirming the result of the present study to some extent. Finally, music therapy has a better effect than the two other interventions on the exercise and sensory functions of patients with AS. Schneider et al. (2010) conducted a recovery training for patients with stroke and found that the recovery training effect of music therapy is better than that of functional exercise training.

After 8 weeks of intervention, the music group has a better effect on the recovery of psychological functions than the painting group, although the effect on the recovery of psychological functions in the music and painting groups is significantly better than the routine treatment group. In particular, music therapy has the best effect on the relief of mental tension and negative emotions caused by AS. This result is consistent with the finding of Tseng et al. (2016), who conducted a meta-analysis of related studies on the use of music therapy to treat patients with schizophrenia. They identified that supporting music therapy is more effective than routine treatment for patients with schizophrenia, especially in reducing patients' negative emotions. Garrido (2015) revealed that even listening to sad music can significantly reduce tension. This finding may be associated with the ability of music to stimulate individuals' adjustment strategy on nonadaptive emotions. Under the influence of negative music, an individual initiates a strategy of enhancing positive emotions to reduce the perception of negative emotions, thereby achieving the purpose of self-protection. Pérez Lloret et al. (2014) explored the relationship between different music types and automated nervous systems and found that individuals who listen to classical music and romantic music have no significant difference in heart rate variability, whereas listening to the music of the new era leads to a significant reduction in heart rate variability. Hence, the impact of music on tension can be achieved by reducing heart rate variability. Second, music therapy has the best effect on improving the positive emotion, cognitive functions, and self-esteem of patients with AS. This result is consistent with the finding of CostaGiomi (2015), who found that childhood music intervention training has a long-term effect on individuals' intelligence and general cognitive ability. Chen (2016) used the random intervention group (music group) and the control group (routine treatment group) to perform three measurements before, during, and after the intervention. The results show that during the intervention and after the intervention, the self-efficacy of the participants in the music group is significantly improved. Some studies have found that the combination of reasonable emotional therapy and music therapy has a better intervention effect on improving college students' self-esteem and psychological flexibility.

\section{CONCLUSION}

A total of 120 patients with AS were randomly divided into the routine treatment group, the painting group, and the music group for 8 weeks of intervention. The intervention effect of music therapy and painting is better than that of routine treatment on patients with AS, and music therapy has the best effect on patients' physical and psychological functions. This finding suggests that music therapy can be applied to provide intervention for patients with AS and to enhance the clinical effect on physical functions and psychological functions. It can also be used as an important auxiliary treatment. The results of this study provide a basis for promoting the clinical application of music therapy. First, music therapy can be used to improve intervention for the physical and mental health of patients with AS. However, its internal mechanism has yet to be explored. Therefore, the clinical application of music therapy can be further enhanced to develop targeted intervention. Second, music therapy is an operative and convenient way that can be combined with other therapies to enhance clinical applications. Third, the correct understanding of music therapy should be enhanced, especially among clinical medical staff, to improve the clinical application of music therapy. 


\section{Acknowledgements:}

This work was supported by Higher Education Quality Project of Anhui Province (2018jyxm0606) and 2019 Quality Engineering Project of Suzhou University (AHSKLW2014D01).

\section{Conflict of interest: None to declare.}

\section{References}

1. Bibb J, Castle D \& Newton R: The role of music therapy in reducing post meal related anxiety for patients with anorexia nervosa. Journal of eating disorders 2015; 3:50

2. Bradt J, Dileo C, Magill L \& Teague A: Music interventions for improving psychological and physical outcomes in cancer patients. Cochrane Database of Systematic Reviews 2016; 8:CD006911

3. Carlson E, Saarikallio S, Toiviainen P, Bogert B, Kliuchko $M$ \& Brattico E: Maladaptive and adaptive emotion regulation through music: a behavioral and neuroimaging study of males and females. Frontiers in human neuroscience 2015; 9:466

4. Chen X, Origasa H, Ichida F, Kamibeppu $K$ \& Varni JW: Reliability and validity of the Pediatric Quality of Life Inventory ${ }^{\mathrm{TM}}\left(P e d s Q L^{\mathrm{TM}}\right)$ Short Form 15 generic core scales in Japan. Quality of Life Research 2007; 16:1239-1249

5. Chen XJ, Hannibal $N \&$ Gold C: Randomized trial of group music therapy with Chinese prisoners: impact on anxiety, depression, and self-esteem. International journal of offender therapy and comparative criminology 2016; 60:1064-1081

6. Costa-Giomi E: The long-term effects of childhood music instruction on intelligence and general cognitive abilities. Update: Applications of Research in Music Education 2015; 33:20-26

7. De Niet $G$, Tiemens B, Lendemeijer B \& Hutschemaekers $G$ : Music-assisted relaxation to improve sleep quality: meta-analysis. Journal of advanced nursing 2009; 65:1356-1364

8. Dundar U, Solak O, Toktas H, Demirdal US, Subasi V, Kavuncu $V$ \& Evcik D: Effect of aquatic exercise on ankylosing spondylitis: a randomized controlled trial. Rheumatology international 2014; 34:1505-1511

9. Fancourt D, Perkins R, Ascenso S, Carvalho LA, Steptoe $A \&$ Williamon A: Effects of group drumming interventions on anxiety, depression, social resilience and inflammatory immune response among mental health service users. PloS one 2016; 11:e0151136

10. Gallego MG \& Garcia JG: Music therapy and Alzheimer's disease: Cognitive, psychological, and behavioural effects. Neurología (English Edition) 2017; 32:300-308

11. Garrido $S$ \& Schubert E: Moody melodies: Do they cheer us up? A study of the effect of sad music on mood. Psychology of Music 2015; 43:244-261

12. Garza-Villarreal EA, Pando V, Parsons C \& Vuust P: Music-induced analgesia in chronic pain conditions: $a$ systematic review and meta-analysis. bioRxiv 2017; 105148

13. Ghetti CM: Active music engagement with emotionalapproach coping to improve well-being in liver and kidney transplant recipients. Journal of music therapy 2011; 48:463-485
14. Hakkou J, Rostom S, Mengat M, Aissaoui N, Bahiri R \& Hajjaj-Hassouni N: Sleep disturbance in Moroccan patients with ankylosing spondylitis: prevalence and relationships with disease-specific variables, psychological status and quality of life. Rheumatology international 2013; 33:285-290

15. Hou J, Song B, Chen AC, Sun C, Zhou J, Zhu H \& Beauchaine TP: Review on neural correlates of emotion regulation and music: implications for emotion dysregulation. Frontiers in psychology 2017; 8:501

16. Kekecs $Z$ \& Varga K: Positive suggestion techniques in somatic medicine: A review of the empirical studies. Interventional Medicine and Applied Science 2013; 5:101-111

17. Kjeken I, Bø I, Rønningen A, Spada C, Mowinckel P, Hagen KB \& Dagfinrud H: A three-week multidisciplinary in-patient rehabilitation programme had positive longterm effects in patients with ankylosing spondylitis: randomized controlled trial. Journal of rehabilitation medicine 2013; 45:260-267

18. Koelsch S: Music $\square$ evoked emotions: principles, brain correlates, and implications for therapy. Annals of the New York Academy of Sciences 2015; 1337:193-201

19. Koelsch S: Investigating the neural encoding of emotion with music. Neuron 2018; 98:1075-1079

20. Lee CH, Lee CY, Hsu MY, Lai CL, Sung YH, Lin CY \& Lin LY: Effects of music intervention on state anxiety and physiological indices in patients undergoing mechanical ventilation in the intensive care unit: a randomized controlled trial. Biological research for nursing 2017; 19:137-144

21. Li ML \& Ren YJ: Intervention Effects of Motivation Interviewing Chinese Modified on the Mental Health of College Students with Exercise Dependence. Psychiatric Quarterly 2019; 90:447-459

22. Loewy J, Stewart K, Dassler AM, Telsey A \& Homel P: The effects of music therapy on vital signs, feeding, and sleep in premature infants. Pediatrics 2013; 131:902-918

23. O'Dwyer T, Monaghan A, Moran J, O'Shea F \& Wilson $F$ : Behaviour change intervention increases physical activity, spinal mobility and quality of life in adults with ankylosing spondylitis: a randomised trial. Journal of Physiotherapy 2017; 63:30-39

24. Yang J, Tang SY, Zhou W: Effect of mindfulness-based stress reduction therapy on work stress and mental health of psychiatric nurses. Psychiatr Danub 2018; 30:189-196

25. Pérez Lloret S, Diez JJ, Domé MN, Alvarez Delvenne A, Braidot N, Cardinali DP, et al: Effects of different" relaxing" music styles on the autonomic nervous system. Noise \& Health 2014; 16:279-284

26. Robarts JZ: The Healing Function of Improvised Songs in Music Therapy with a Child Survivor of Early Trauma and Sexual Abuse. In Hadley S (ed): Psychodynamic Music Therapy: Case Studies, 141-182. Gilsum, NH, 2003

27. Schneider S, Müünte T, Rodriguez-Fornells A, Sailer $M \&$ Altenmü̈ller E: Music-supported training is more efficient than functional motor training for recovery of fine motor skills in stroke patients. Music Perception: An Interdisciplinary Journal 2010; 27:271-280

28. Leleikiene A, Reklaitiene D, Pozeriene J: Physical Activity is a Good Way to Restore Work Productivity of People with Depression. Transformations in Business \& Economics 2018; 17(2A):499-510

29. Tseng PT, Chen YW, Lin PY, Tu KY, Wang HY, Cheng YS, et al: Significant treatment effect of adjunct music therapy 
to standard treatment on the positive, negative, and mood symptoms of schizophrenic patients: a meta-analysis. BMC psychiatry 2016; 16:16

30. Thompson WF: Music, thought, and feeling: Understanding the psychology of music. Oxford University Press, 2015

31. Tumuluri I, Hegde $S$ \& Nagendra HR: Effectiveness of music therapy on focused attention, working memory and stress in Type 2 diabetes: An exploratory study. International journal of yoga 2017; 10:167-170

32. Van Lith T: Art therapy in mental health: A systematic review of approaches and practices. The Arts in Psychotherapy 2016; 47:9-22

33. Wang CF, Sun YL \& Zang HX: Music therapy improves sleep quality in acute and chronic sleep disorders: $A$ meta-analysis of 10 randomized studies. International Journal of Nursing Studies 2014; 51:51-62

34. Wu B: Influence of a Music Therapy Program to Prevent Somatic Symptom Disorder Pain: An Experimental Study. NeuroQuantology 2017; 15:158-163

35. Wu JJ, Penfold RB, Primatesta P, Fox TK, Stewart C, Reddy SP, et al: The risk of depression, suicidal ideation and suicide attempt in patients with psoriasis, psoriatic arthritis or ankylosing spondylitis. Journal of the European Academy of Dermatology and Venereology 2017; 31:1168-1175
36. Xue J, Zhang LY \& Yang Q: Music therapy to alleviate nausea and vomiting and improve quality of life in cancer patients after chemotherapy. Journal of Nursing 2017; 24:70-72

37. Yllmaz Ö, Tutoğlu A, Garip Y, Özcan E \& Bodur H: Health-related quality of life in Turkish patients with ankylosing spondylitis: impact of peripheral involvement on quality of life in terms of disease activity, functional status, severity of pain, and social and emotional functioning. Rheumatology international 2013; 33:1159-1163

38. Zagar I, Delimar V, Cota S, Peric D, Laktasic-Zerjavic N, Peric P: Correspondence of vitamin D status with functional scores and disease activity among Croatian patients with ankylosing spondylitis: a preliminary study. Psychiatr Danub 2019; 31(suppl 1):105-111

39. Zeng Y, Yang Y, Liu RT \& Yang J: On the Function of Music Therapy in Treating Physical and Psychological Disease. Genomics and Applied Biology 2015; 34:1575-1578

40. Zhang LD, Yang H, Liu CM, Gao D, Xia XM \& Guo Y: Clinical Application of Wuxing Music Therapy in Improving Anxiety and Depression Symptoms of Patients with Advanced Cancer. Chinese General Practice Nursing 2018; 16:80-81

41. Zhao HY \& Wang Y: The Application of Expressive Writing in Mental Health Education of Higher Vocational Schools. Vocational and Technical Education 2011; 32:74-78

Correspondence:

Yuan Qin, MD

Conservatory of Music, Suzhou University

Suzhou, Anhui, 234000 China

E-mail:qqinyuan@yeah.net 